mortality was 12.5 percent (mean follow-up duration 5.46 years). Conclusions Data analysis is ongoing with plans to compare relevant patient demographics and outcomes, and to analyse questionnaires to elucidate how surgeons incorporate fMRI data into their therapeutic approach.

PC3 - 191

doi:10.1017/cjn.2016.387

\section{Assessment of Preoperative Functional MRI Measurement of Language Lateralisation in Brain Tumour Patients}

E. Kosteniuk ${ }^{I}$, J.C. Lau, J.F. Megyesi

${ }^{1}$ School of Medicine and Dentistry, University of Western Ontario, London, ON skosteniuk2018@meds.uwo.ca

This study aims to evaluate reliability of clinical functional magnetic resonance imaging (fMRI) in identifying language lateralisation index (LI), verified with Edinburgh handedness inventory (EHI), in brain tumour patients. Methods In this retrospective study, 31 of a single surgeon's brain tumour patients over a 12 year period have been selected. Lesion type varied, 12 (39 percent) were high grade gliomas, 10 (32 percent) low grade gliomas, 3 (10 percent) meningiomas, and 6 (19 percent) other types. Patients underwent language fMRI paradigms for preoperative assessment, and a neuroimaging analyst was able to identify an LI value for at least one Brodmann area (BA). For each paradigm, a neuroimaging analyst attempted to calculate LI for Wernicke's area (BA 22) and Broca's area (BA 44 and 45). Results Of 113 total LI values, 66 (58 percent) were concordant to EHIpredicted hemispheric dominance. Reliability of language LI appears dependent upon the type of language task performed. Verb generation correctly identified Broca's area in 18 patients (64 percent) and Wernicke's area in 11 patients (61 percent), sentence completion correctly identified Broca's area in 18 patients (72 percent) and Wernicke's area in 9 patients (60 percent), and naming correctly identified Broca's area in 7 patients (47 percent) and Wernicke's area in 3 patients (27 percent). Conclusions Results show limited correlation between language LI determined by fMRI and EHI. The main limitation of this study is that language LI is being compared to EHI, rather than gold standard measure of hemispheric dominance (e.g. Wada).

\section{OTHER CLINICAL}

PC3 - 128

doi:10.1017/cjn.2016.388

Clinical Profile and Treatment Outcomes of Patients with Primary CNS Lymphoma in a Tertiary Hospital in the Philippines: An Eight-Year Retrospective Review

\section{M.C. Concepcion Sales ${ }^{I}$ \\ ${ }^{I}$ Philippine General Hospital, Manila, Philippines macarmelamd@yahoo.com}

Primary CNS Lymphoma (PCNSL) is an unusual extranodal form of Non-Hodgkin's lymphoma with a locally aggressive course but a rare tendency to disseminate systemically. It has been documented in that the clinical characteristics and response to treatment among Asians is comparable to the Western population yet no studies done locally are available. Objectives: This study aims to determine the clinico-pathologic profile of patients diagnosed with PCNSL seen at Philippine General Hospital (PGH) from January 2006 to September, 2014 and to evaluate the patients' response to the following treatment modalities: 1) Combination chemotherapy 2) Chemo-RT 3) Single agent chemotherapy and 4) no specific anti-lymphoma treatment. Methodology: This is a descriptive and retrospective study that included all cases of histologically-proven PCNSL seen at the PGH from January 2006 to September, 2014. The clinical profile, imaging studies and biopsy findings were obtained from the patient records. The survival rates at the end of one and two years of diagnosis were computed. Results and Conclusion. Among patients diagnosed with PCNSL at PGH, there is a higher incidence of PCNSL among males with a male to female ratio of 1.4:1 and have a younger onset with a median age of 50.2 years. Most patients presented with signs of increase ICP and majority had solitary cortical lesions with histopathologic diagnosis of diffuse large B cell lymphoma. Patients who did not undergo any form of treatment had a mean survival of 10 months. Immunocompromised patients had a shorter life-span with a mean survival of 7.5 months. Treatment of combination chemotherapy with HD-MTX and Rituximab had the most favorable outcome followed by HD-MTX only with a 2 year survival rate of $100 \%$ and $66 \%$ respectively while patients who underwent chemo-RT had a 2 year survival rate of $33 \%$ with a high incidence of neurocognitive delay.

PC3 - 129

doi:10.1017/cjn.2016.389

Primary Intracranial Round Cell Sarcoma in an HIV Patient: A Case Report and a Review of Literature

\section{M.C. Concepcion Sales ${ }^{l}$ \\ ${ }^{I}$ Philippine General Hospital, Manila, Philippines macarmelamd@yahoo.com}

Primary intracranial sarcoma among patients diagnosed with HIV is rare. Case reports published have shown that there is an increasing number of cases of leiomyosarcoma, hemangiopericytoma and rhabdomyosarcoma among these patients. Further, there are also few reports that sarcomas may present atypically, sometimes mimicking a brain abscess. We report a case of a 26 year old male, newly diagnosed HIV, who presented with bilateral chronic suppurative otitis media associated fever, headache and vomiting. Neuroimaging showed with multiple rim enhancing masses on the left temporal, parietal and occipital areas and bilaterally sclerosed mastoid air cells. Initial impression was an otogenic abscess. Burrhole craniotomy and evacuation of the cystic masses was done. Histopathologic examination revealed a small round blue cell sarcoma. Different immunostains were done to differentiate the various subtypes of sarcomas possible. Patient was discharged improved but did not consent to chemotherapy or radiotherapy.

PC3 - 130

doi:10.1017/cjn.2016.390

A Meta-Analysis on the use of High-Dose Methotrexate Only Versus Combination Chemotherapy for the Treatment of Newly-Diagnosed Patients with Primary CNS Lymphoma 